\section{Excepciones al uso del consentimiento informado en investigación: ¿cuándo es esto posible en Chile?}

\author{
CAROL ZÚÑIGA' 1,2,a, JESSICA ZÚÑIGA-HERNÁNDEZ ${ }^{3, \mathrm{~b}}$
}

\section{Exception to informed consent in research: when is this possible in Chile?}

\begin{abstract}
Exceptions or waivers to informed consent in research in Chile are an ethical issue that has not been addressed in all its complexity by the scientific community. The possible waivers to this process could make the difference for the feasibility, success or failure of a study. The purpose of this document is to clarify within the available information, what is the current situation about the exceptions to informed consent in research and what are the ethical-legal guidelines in Chile. Articles were reviewed in both English and Spanish from indexed journals and those documents that meet the inclusion criteria were selected. There is limited information available about waivers to informed consent, which indicates that the ethical guidelines are similar among countries that honor the Declaration of Helsinki, including Chile. However, the current Chilean legislation does not include exceptions. Ethics Committees that authorize research in line with international ethical guidelines could allow possible exceptions. Based on our findings, it is necessary to evaluate possible exceptions for informed consent for research performed in Chile, both from the legal point of view and based on the experience of scientists.
\end{abstract}

(Rev Med Chile 2019; 147: 1029-1035)

Key words: Bioethical Issue; Ethics; Informed Consent; Research Subjects.

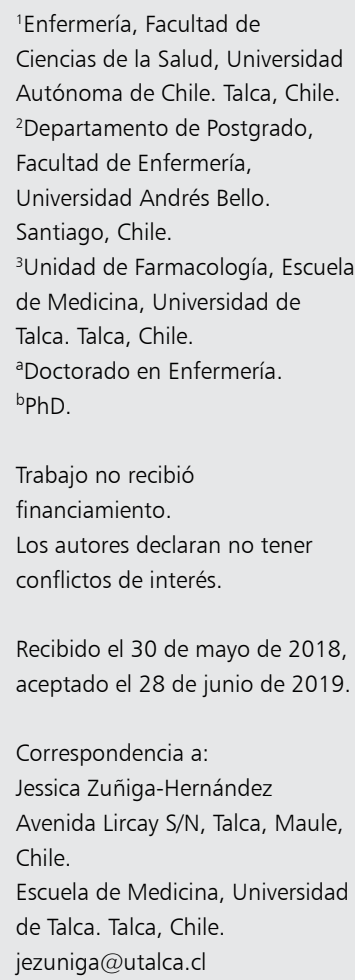

\section{Aproximaciones conceptuales}

E 1 Consentimiento Informado (CI) es un proceso ético-práctico imprescindible en la operacionalización de estudios científicos: tiene un sustento ético y legal conocido por quienes realizan tareas de investigación. Un consentimiento se entiende como un medio de comunicación entre el investigador y la persona atendida este debe ser libre e informado, requiriendo que la persona comprenda la información contenida. Sin el consentimiento adecuado, toda actividad sanitaria y/o científica puede llegar a vulnerar los derechos fundamentales del paciente, como el derecho a la vida, la libertad y la propiedad ${ }^{1}$. Históricamente han ocurrido a nivel mundial, situaciones en las cuales ha sido necesario admitir ensayos de investigación en ausencia del consentimiento directo del sujeto, y es a partir de estas situaciones que nace la necesidad de discutir en torno al tema de las renuncias del CI, básicamente en cuanto a sus excepciones, exenciones y dispensas. Junto a lo anterior se debe considerar que la práctica médica y los ensayos científicos pueden conllevar un riesgo en sí mismos (considerando el riesgo como una variable epidemiológica equivalente a efecto, probabilidad de ocurrencia de una patología en una población determinada, expresado a través del indicador paradigmático de incidencia ${ }^{2,3}$ ).

El propósito de este documento es esclarecer lo que la literatura nos muestra en cuanto a las excepciones al consentimiento informado en la 
investigación, en qué casos es esto posible y cuando pudiera justificarse dicha excepción en Chile (excluyendo aquellos estudios, independiente del diseño que no necesitan contar con autorización del sujeto estudiado dado que no contemplan el uso de datos sensibles o estos se encuentran bajo anonimato completo). El objetivo entonces es determinar en qué casos se puede hacer excepciones al CI en investigación con seres humanos avalados en el marco regulatorio de nuestro país, en base a una revisión bibliográfica y una reflexión desde los aspectos éticos involucrados en el tema, buscando aportar al esclarecimiento de situaciones que pudieran llevar a confusión a un investigador en su propósito de optimizar la relación riesgo beneficio como resultado de un estudio.

\section{Metodología de búsqueda}

Se realizó una búsqueda bibliográfica en las bases de datos PubMed, Web of Science, Scopus, y Scielo. Los términos utilizados fueron "exception to/from informed consent", "exception without consent", "consent waiver" y su relación con "research" y/o "ethics" (tanto en inglés como en español). La búsqueda fue circunscrita a artículos asociados con códigos internacionales de ética, organizaciones que contuviesen información respecto a investigación sin el CI, aspectos legales, éticos, excluyendo casos particulares (emergencia, incapacidad temporal y estudios observacionales). También fueron incluidos en esta revisión informes como el código de Núremberg, la declaración de Helsinki, el informe Belmont, el CIOMS, y leyes constitucionales chilenas. Todo artículo que describe solo el CI sin dispensas fue excluido.

Esta revisión ha permitido observar una variedad de artículos referentes al uso y excepciones al CI en la práctica clínica. Evidenciándose que este tema está mucho más estudiado desde la perspectiva ético-clínica que en investigación, considerando las responsabilidades médico/ paciente involucradas y las eventuales repercusiones de dichas excepciones ${ }^{4}$. Existen evidentes diferencias entre ambas prácticas, aun cuando se pueda consensuar que ambas tributan al respeto de los principios éticos y buscan la protección de los individuos sujeto de atención ${ }^{5}$. Sin embargo, esta diferencia entre $\mathrm{CI}$ en la práctica clínica y en la práctica científica en cuanto a la cantidad de evidencia disponible, nos da un primer indicio de cuanto y como se ha relevado la temática en cuanto a excepciones se refiere.

A partir de los lineamientos éticos internacionales emanados del código de Núremberg primero $^{6}$, y de la declaración de Helsinki y del Reporte Belmont después ${ }^{7,8}$, se han definido con relativa claridad los elementos que deben cautelarse en investigaciones que involucren seres humanos a lo largo de todo el mundo. Se debe recordar que la declaración de Helsinki formalizó algunos principios enunciados en Nuremberg y se ha convertido en un referente internacional para la creación de instituciones destinadas a la protección de sujetos humanos y difusión de principios éticos en la ciencia. Sin embargo, esta declaración no es jurídicamente vinculante para sus signatarios, entre ellos Chile, por lo que las naciones son libres de crear sus propias formas de aplicación de los principios mencionados siempre que exista coherencia con los principios planteados en dicha declaración ${ }^{1}$.

\section{Principios éticos}

Evidentemente, los principios éticos de Respeto, Beneficencia y Justicia han querido ser cautelados a partir de este respaldo teórico consensuado y que viene a ser el referente en cuanto a protección de los sujetos de estudio se refiere. Recordemos que el respeto por las personas guarda relación con los requisitos morales ${ }^{9}$ que responden al reconocimiento de la autonomía personal de los participantes de un estudio y de resguardar con especial atención a las personas mas vulnerables. El reconocimiento de la autonomía se evidencia en la capacidad de deliberación personal acerca de los propios deseos y necesidades y el actuar en concordancia con estos. Además, esta capacidad de deliberación puede verse alterada por factores intrínsecos o extrínsecos, dados por situaciones circunstanciales o definitivas y que merman la posibilidad de efectuar elecciones de vida. Esto puede darse por enfermedad, discapacidad o restricción de la libertad, por ejemplo. En cuanto al principio de Beneficencia, Riquelme y Abreu $(2016)^{10}$ indican que este se refiere a "la obligación de actuar en beneficio de otros, promoviendo sus legítimos intereses y suprimiendo prejuicios." y "... de este principio de beneficencia se deriva la obligatoriedad de informar de los posibles riesgos y beneficios de la investigación. Por tanto, deberá haber una relación riesgo/beneficio favorable para 
el sujeto". Los aspectos éticos en cuanto a Justicia en investigación se refieren principalmente al deber ético de tratar a cada persona de acuerdo con lo que le corresponde. Según Mancini Rue$\mathrm{da}^{11}$ en investigación es especialmente aplicable el principio aristotélico de la justicia distributiva, que exige la distribución equitativa tanto de los costos como de los beneficios de la participación en actividades de investigación. La equidad puede ser vista de distintas maneras, pero parece más cercana a nuestra visión solidaria de la sociedad el "dar a cada cual según su necesidad". Las diferencias que puedan ocurrir en esa distribución se justifican sólo si se basan en distinciones que sean pertinentes desde el punto de vista moral, como lo es la vulnerabilidad ${ }^{11}$.

Sin lugar a dudas, los principios éticos han sido una guía imponderable en cuanto a la práctica de la investigación, en virtud de los hechos históricos acontecidos en ausencia de ellos o en cuanto estos no eran exigibles universalmente en el ámbito científico ${ }^{12}$. Pero aun cuando hoy estos principios son exigidos en la mayoría de los países que generan investigaciones científicas, existen intereses públicos y/o factores especiales que pudiesen "exceptuar" o "eximir" de la necesidad de solicitar el consentimiento informado, a pesar de ser derivada de estos principios éticos

\section{Excepción al consentimiento informado: Resultados}

\section{Situación Internacional}

Específicamente en cuanto al respeto a la autonomía de las personas pareciese que al aplicar el proceso de CI en investigación en personas, gran parte del deber ético se encuentra cubierto. Sin embargo, de acuerdo a la Declaración de Helsinski ${ }^{7}$ en sus puntos 29, 30 y 32 los posibles sujetos de investigación que no pudieren otorgar su CI para participar en un estudio, deben ser representadas en este derecho por alguien significativo para estos efectos. Sin embargo, si este representante o tutor no es posible de ser hallado y la investigación debe de ser realizada en sujetos que no puedan otorgar dicho consentimiento (pacientes inconscientes, personas incapacitadas mentales severas, personas en situación de emergencia vital, etc.) este puede ser dispensado si la situación antes descrita fue considerada en el protocolo de estudio y aprobada por un comité de ética pertinente. Incluso, el punto 32 particularmente se refiere a que "Para la investigación médica que utiliza material o datos humanos identificables, como la investigación de material o datos contenidos en biobancos o repositorios similares, los médicos deben solicitar el CI para su recopilación y almacenamiento y/o reutilización. Puede haber situaciones excepcionales en las que el consentimiento sería imposible o impracticable para obtener para tal investigación. En tales situaciones, la investigación puede realizarse solo después de la consideración y aprobación de una investigación Comité de Ética”. Por otra parte, las Pautas Éticas Internacionales Para la Investigación Relacionada con la Salud con Seres Humanos ${ }^{13}$ (CIOMS)-2017 dedican una pauta completa a la temática de discusión: la Pauta 10 CIOMS, sobre "modificaciones y dispensas del consentimiento informado". Se aclara en esas líneas, que ninguna investigación debe ser comenzada sin la obtención del CI de sus participantes o sus tutores legales, "a menos que (los investigadores) hayan recibido la aprobación explícita de un Comité de Ética de la investigación". Resalta que antes de conceder una dispensa al proceso del consentimiento "los investigadores y los Comités de Ética de la investigación deberían primero procurar determinar si podría modificarse el CI de una manera que preserve la capacidad del participante para comprender la naturaleza general de la investigación y decidir si participa”.

Esta pauta aclara las condiciones en que un comité de ética pudiera aprobar una modificación o una excepción al CI en una investigación. Estos requisitos de la investigación son: i) La Investigación no sería factible o viable sin dicha exención; ii) La investigación tiene un valor social importante y iii) Entraña apenas riesgos para los participantes. Por lo anterior, la dispensa del consentimiento acontecería solo sí el comité de ética está convencido de que la investigación cumple con estos requisitos y siempre que se ciña a los preceptos legales locales (denominados "otras disposiciones"). La presentación de estas justificaciones siempre dependerá del investigador interesado ${ }^{13}$.

En 1996 la Administración de Medicamentos y Alimentos de los Estados Unidos (FDA) generó una regulación federal relativa a los ensayos clínicos que utilizan exenciones al consentimiento informado (EFIC), donde es posible observar que las 
dispensas asociadas a investigación en emergencias están recomendadas para poblaciones vulnerables, es decir sujetos que no tienen capacidad de dar su consentimiento a tiempo (situación de emergencia) y requieren además obtener autorización legal de un tutor o representante. Para proteger a estas personas vulnerables se debe seguir el marco regulatorio propuesto por FDA en 1996 y actualizado en $2013^{14,15,16}$. Esta guía además posee una garantía extra de protección al sujeto que incluye "consultas a la comunidad" y "divulgación pública" antes de iniciada una investigación ${ }^{14}$. Si bien esta regulación es solo para investigaciones en territorio estadounidense, la FDA es considerada un referente internacional y por ende esta regulación podría ser utilizada como una guía para el desarrollo de investigaciones en ensayos clínicos.

\section{Situación chilena actual}

En nuestro país existen a la fecha dos Leyes de la República que versan sobre la investigación en general y sobre el CI en investigación en particular. Este marco legal hace referencia a la obligatoriedad de la obtención del CI en toda investigación en seres humanos y respecto de la necesidad de un representante legal en caso de que individuo de estudio no esté en condiciones mentales para otorgar su autorización. La Ley 20.120 "Sobre la investigación científica en el ser humano, su genoma, y prohíbe la clonación humana" en vigencia desde el año 2006, legisla sobre los aspectos ético-prácticos de la investigación en seres humanos y su objetivo final pretende proteger la vida de los mismos desde el momento de la concepción en su integridad física y psíquica, como en su diversidad e identidad genética ${ }^{17}$. En su artículo no 11 , especifica que "toda investigación científica en un ser humano deberá contar con su consentimiento previo, expreso, libre e informado, o en su defecto, el de aquel que deba suplir su voluntad en conformidad a la Ley". Esto deberá hacerse a través de un acta firmada por la persona a consentir, el director responsable de la investigación y el director del establecimiento donde dicha investigación será llevada a cabo. No existe, según esta declaración excepciones legales a este proceso.

Para complementar el respaldo legal y la protección de los ciudadanos en cuanto a investigación y producción científica, el estado de Chile ha promulgado en el año 2012 la Ley no 20.584 que "Regula los derechos y deberes que tienen las personas en relación con acciones vinculadas a su atención en salud"18 la que en el párrafo $n^{\circ} 7$ "De la protección de la autonomía de las personas que participan en una investigación científica" es muy explícita en su artículo $n^{\circ} 21$ sobre la exigibilidad del CI como requisito para participar en una investigación respaldándose a su vez en la Ley 20.120 , toda vez que en su artículo no 28 aclara que "ninguna persona con discapacidad psíquica o intelectual que no pueda expresar su voluntad podrá participar en una investigación científica". Se agrega que quienes tengan estas discapacidades, pero puedan manifestar su voluntad de participación, aún deben contar con la autorización de un comité de ética para su incorporación como sujeto de estudio en una investigación. Nuevamente, no se observa pronunciamiento en cuanto a las potenciales excepciones a la Ley ${ }^{19}$, incluso cuando se contemplan casos en los que no se pueda dar consentimiento.

Derivado de la misma Ley 20.120 se han creado Comités de Ética acreditados bajo las normas internacionales dictadas por la $\mathrm{OMS}^{20}$ y que buscan precisamente cautelar los derechos y la protección de los individuos que participen en investigaciones en Chile. Estos comités deben regirse por los aspectos legales ya mencionados, respaldando su accionar ético basados en las normas internacionales que se referenciaron más arriba ${ }^{21,22}$.

En el año 2017, en Chile se publica un análisis normativo respecto al "Acceso excepcional a las fichas clínicas sin consentimiento informado, con fines de investigación" por la Comisión Ministerial de Investigación en salud- $\mathrm{CMEIS}^{23}$, en donde se clarifica una situación particular: el eventual acceso a la información que existe dentro de las fichas clínicas de los usuarios del sistema de salud chileno para efectos de investigación, que en rigor requerirían la autorización de las personas "dueñas” de la información contenida. Al respecto, el informe indica "Existe el acceso sin consentimiento del titular respecto de determinadas instituciones autorizadas por la ley, entre éstas el Ministerio de Salud en ejercicio de sus funciones en la investigación en áreas determinadas con fines de salud pública" Agregando que "El acceso a la ficha clínica con fines de investigación científica no fue enfrentado en definitiva por la Ley ${ }^{\circ} 20.584$ sobre Derechos y Deberes y corresponde hacerlo sobre la base de la Ley de Investigación $\mathrm{n}^{\circ} 20.120$, requiriendo por lo tanto el consentimiento in- 
formado específico" y que "El uso del contenido de las Fichas Clínicas para fines de investigación, como toda investigación científica con seres humanos, requiere la aprobación de un Comité Ético Científico acreditado", además de la autorización del Director del establecimiento donde se realiza el estudio quien es el garante de la custodia de las fichas clínicas y será a quien le corresponda resguardar la información sensible que ella pudiera contener. En el caso de información que esté anonimizada, no hay inconveniente para su uso con fines investigativos.

El comité de ética de investigación en seres humanos (CEISH) de la Escuela de Medicina de la Universidad de Chile, en el año 2014, contempla la dispensa del CI para investigaciones retrospectivas si la información a recopilar está en una base de datos anónima, las que no se asocian a un titular

Tabla 1. Estudios de las dispensas del consentimiento informado

\begin{tabular}{|c|c|c|c|}
\hline Año & Revista & Primer autor & Excepciones \\
\hline 2003 & $\begin{array}{l}\text { Revista Mexicana de } \\
\text { Bioética (México) }\end{array}$ & $\begin{array}{l}\text { FeinHolz-Klip J. } \\
\text { (México) }^{25}\end{array}$ & $\begin{array}{l}\text { Propone criterios para aceptación de dispensas: 1) Riesgo mínimo; 2) La } \\
\text { dispensa no afecta el bienestar y los derechos de los pacientes; 3) Sin la } \\
\text { dispensa no se podría realizar el estudio; 4) Se proporciona información } \\
\text { pertinente a los participantes al finalizar el estudio }\end{array}$ \\
\hline 2003 & Stroke (EEUU) & $\begin{array}{l}\text { Bateman BT. } \\
\quad(\text { EEUU })^{26}\end{array}$ & $\begin{array}{l}\text { Menciona } 12 \text { criterios aditivos en las cuales se justifica las dispensas en } \\
\text { investigación relacionada al accidente cerebrovascular y la emergencia } \\
\text { que impide obtener apropiadamente el } \mathrm{Cl}\end{array}$ \\
\hline 2007 & $\begin{array}{l}\text { Academic Emergency } \\
\text { Medicine (EEUU) }\end{array}$ & $\begin{array}{l}\text { Triner W. } \\
(\text { EEUU })^{16}\end{array}$ & $\begin{array}{l}\text { Analiza la actitud de la población frente a las dispensas en investigación } \\
\text { médica de urgencias. La población estudiada acepta la necesidad de } \\
\text { investigación y las dispensas, pero no se comprometen a participar en } \\
\text { dichas investigaciones, además se detecta un bajo entendimiento de los } \\
\text { riesgos y beneficios del estudio en la población analizada }\end{array}$ \\
\hline 2014 & $\begin{array}{l}\text { Revista de Parasitología } \\
\text { Tropical (India) }\end{array}$ & $\begin{array}{l}\text { Mandal J. } \\
(\text { India })^{27}\end{array}$ & $\begin{array}{l}\text { Analiza la situación en la India, donde existen posibles excepciones al Cl } \\
\text { en investigación que aluden a los lineamientos legales y de ética local }\end{array}$ \\
\hline 2015 & $\begin{array}{l}\text { Revista de la Facultad } \\
\text { de Ciencias Médicas } \\
\text { (Argentina) }\end{array}$ & $\begin{array}{l}\text { Kenneth V. } \\
(\text { EEUU })^{28}\end{array}$ & $\begin{array}{l}\text { Revisa la exención del } \mathrm{Cl} \text { en situaciones de emergencia cuando "la situa- } \\
\text { ción de estudio no dará tiempo para que los participantes potenciales } \\
\text { de la investigación se involucren apropiadamente en el proceso de Cl, } \\
\text { cuando los tratamientos pruebas o dispositivos solo pueden ser usados en } \\
\text { situaciones de emergencia y cuando la situación puede poner en peligro } \\
\text { la capacidad de los participantes potenciales y sus representantes para } \\
\text { tomar decisiones informadas debido a la ansiedad el dolor o la alteración } \\
\text { de conciencia". }\end{array}$ \\
\hline 2016 & $\begin{array}{l}\text { BMC Medical Ethics } \\
\text { (Países Bajos) }\end{array}$ & $\begin{array}{l}\text { Rebers S. } \\
\text { (Países Bajos) }^{29}\end{array}$ & $\begin{array}{l}\text { Revisa la exención de los } \mathrm{Cl} \text {, generando } 4 \text { posibles razones para ella: 1) La } \\
\text { disminución de la validez y calidad de los datos; 2) Angustia o confusión } \\
\text { de los participantes; 3) Problemas prácticos; 4) Razones éticas de renuncia } \\
\text { al consentimiento }\end{array}$ \\
\hline 2016 & $\begin{array}{l}\text { Journal of General } \\
\text { Internal Medicine } \\
\text { (EEUU) }\end{array}$ & $\begin{array}{l}\text { Flory J. } \\
(\text { EEUU })^{30}\end{array}$ & $\begin{array}{l}\text { Revisa los usos del } \mathrm{Cl} \text { y sus exenciones, donde se observan: i) diseños } \\
\text { aleatorios por conglomerados y ii) investigación de emergencia, usan de } \\
\text { forma rutinaria la dispensa al } \mathrm{Cl} \text {, con la justificación de que el consenti- } \\
\text { miento informado no es factible en esos entornos y en estudios aleatorios } \\
\text { de bajo riesgo ejecutados } \sin \mathrm{Cl}\end{array}$ \\
\hline 2017 & $\begin{array}{l}\text { Revista Española de } \\
\text { Medicina Legal (España) }\end{array}$ & $\begin{array}{l}\text { Bezhold G. } \\
(\text { España) })^{31}\end{array}$ & $\begin{array}{l}\text { Analiza la realidad española: "la legislación española no contempla ex- } \\
\text { cepciones al Cl para acceder a su ficha clínica" }\end{array}$ \\
\hline 2017 & Acta Bioethica (Chile) & $\begin{array}{l}\text { Frunza A. } \\
\text { (Rumania) }^{32}\end{array}$ & $\begin{array}{l}\text { Revisa los diversos formatos de Consentimientos informados utilizados en } \\
\text { la atención clínica en la realidad de Rumania. Se describen aspectos éticos } \\
\text { y legales que conforman el marco de acción para esta temática y se dis- } \\
\text { cute respecto de lo ético que puede ser la utilización del consentimiento } \\
\text { generado de la práctica clínica para realizar posteriores investigaciones } \\
\text { médicas con datos o muestras biológicas recolectadas para otro fin }\end{array}$ \\
\hline
\end{tabular}


con identidad o identificable (no vinculable) y por ende no es necesario contactar a las fuentes, ya que no constituyen datos sensibles o confidenciales. Para otorgar esta dispensa "el CEISH debe considerar el cumplimiento de los siguientes criterios: a) confidencialidad de la declaración; b) asegurar el anonimato del paciente (encriptación de datos identificadores); c) responsabilidad/compromiso del investigador; d) valor y validez científica de la investigación; e) justificación social y científica de la investigación propuesta" 24 .

\section{Situaciones internacionales}

Existen algunas fuentes de información adicionales que permiten verificar dispensas del CI, estos artículos buscan alinear criterios para la aceptación de renuncias a nivel local, recabar información sobre las excepciones que se han realizado en el ámbito de investigación y analizar los lineamientos legales (Tabla 1).

\section{Consideraciones finales}

Según los hallazgos en el marco ético vigente para nuestro país, es el Comité de ética donde se presenta el estudio quien debe asegurar la protección de los derechos y el bienestar de los seres humanos que participan en los ensayos clínicos y eventualmente, en el marco de las norma y leyes que rigen su rango de decisión, pudiesen autorizar si éticamente puede ser realizada una investigación sin contar con el CI de los potenciales sujetos de estudio. Esto es posible solo si se enmarca en las exigencias éticas presentadas en las pautas éticas internacionales (CIOMS, siglas del inglés Council for International Organizations of Medical Sciences) o en las recomendaciones nacionales de la Comisión Ministerial de Ética de la Investigación en Salud (CMEIS) y si se presenta un sustento contextual sólido que resguarde a las personas y justifique la imprescindibilidad de realizar la investigación con esos datos o sujetos de estudio en particular. Se sobreentiende que existe la obligación moral por parte del investigador que lidera el estudio de no transgredir otros aspectos éticos, de generar las condiciones prácticas congruentes y los resguardos tendientes a esta protección. Estos resguardos pudieran incluir el manejo de datos sensibles en bases de datos codificadas, el manejo de los datos recolectados por un investi- gador diferente de quien los recolectó, el manejo estrecho de los posibles riesgos atribuibles a la participación en la investigación y la declaración de parte del investigador líder para hacerse cargo de los posibles daños como consecuencia de dicha participación, entre otras.

Finalmente, si bien la Ley chilena no contempla excepciones al CI, basados en las pautas internacionales de bioética a las que se encuentran adscritos nuestro Comités de Ética acreditados a nivel nacional y a las recomendaciones de la CMEIS, pudiera autorizarse un protocolo de investigación en situación donde se dispense el CI siempre y cuando esta investigación cumpliera con lo siguiente: i) no fuera factible o viable sin dicha exención; ii) la investigación tuviera un valor social importante $y$; iii) esta entrañara apenas riesgos para los participantes. Además, la realización del estudio sin el CI estará siempre sujeta a la aprobación de un comité de ética científica acreditado y a la autorización del director del establecimiento donde eventualmente se realizare el estudio.

\section{Referencias}

1. Koepsell D, Ruiz de Chávez M. Ética de la investigación, integridad científica. Comisión Nacional de Bioética (CONBIOÉTICA)/ Secretaria de Salud, México. 2015; lera edición.

2. Filho NA, Castiel LD, Ayres JR. Riesgo: concepto básico de la epidemiología. Salud Colectiva 2009; 5 (3): 1-23.

3. Guzmán F, Arias C. El concepto de riesgo en medicina. Revista de Fac Med 2011; 19 (2): 241-7.

4. Alfaro L, Magaña M. Realidades conceptuales del consentimiento informado para la seguridad del médico y del paciente. Rev Inst Nal Enf Resp 2008; 21 (3): 21320.

5. Garbus P, Solitario R, Solkiner A. Aspectos éticos en investigaciones no clínicas en el campo de la salud: algunas consideraciones del consentimiento informado en personas declaradas incapaces. Anuario de investigaciones Facultad de psicología UBA 2009; 16: 329-38.

6. Código de Núremberg. Rev Filosofía Univ Costa Rica 2006; XLIV (111-112): 189-90.

7. Asociación Médica Mundial. Principios éticos para la Investigaciones médicas en seres Humanos. Declaración de Helsinski. 64 ${ }^{\circ}$ Asamblea General, Fortaleza, Brasil 2013.

8. National Commission for the Protection of Human Subjects of Biomedical and Behavioral Research. The 
Belmont Report. Washington DC: US Government Printing Office, 1979.

9. Opazo CH. Ética en Investigación: Desde los Códigos de Conducta hacia la Formación del Sentido Ético. Rev Iberoamericana REICE 2011; 9 (2): 61-78.

10. Riquelme I, Álvarez S, Ramos RV, Saborido ML, González HZ. Breves consideraciones sobre la bioética en la investigación clínica. Revista Cubana de Farmacia 2016; 50 (3): ISSN 1561-2988.

11. Mancini H. Normas éticas para la Investigación Clínica. Centro Interdisciplinario de Estudios en bioética. Columna Dr. Roberto Mancini Rueda Universidad de Chile, año consulta 2018. http://www.uchile.cl/ portal/investigacion/centro-interdisciplinario-de-estudios-en-bioetica/publicaciones/76992/normas-eticas-para-la-investigacion-clinica.

12. Michaud CP. Lo ético y lo no ético en investigación clínica. Rev Chil Cardiol 2015; 34 (1): 58-62.

13. Organización Panamericana de la Salud y Consejo de Organizaciones Internacionales de las Ciencias Médicas CIOMS/OMS. Pautas éticas internacionales para la investigación relacionada con la salud con seres humanos, Cuarta Edición. Ginebra 2016: Consejo de Organizaciones Internacionales de las Ciencias Médicas (CIOMS).

14. U.S. Code of Federal Regulations. 21 CFR 50.24: protection of human subjects; informed consent-FDA; final rule. Fed Regist 1996; 61: 51498-533.

15. Klein L, Moore J, Biros M. A 20-year Review: The Use of Exception From Informed Consent and Waiver of Informed Consent in Emergency Research. Acad Emerg Med 2018; 25: 1169-117.

16. Triner W, Jacoby L, Shelton W, Burk M, Imarenakhue S, Watt J, Larkin G, McGree G. Exception from informed consent enrollment in emergency medical research: attitudes and awareness. Acad Emerg Med 2007; 14: 187-91.

17. Biblioteca del Congreso Nacional de Chile. Gobierno de Chile. Ley 20.120. https://www.leychile.cl/Navegar?idNorma $=1039348$.

18. Biblioteca del Congreso Nacional de Chile. Gobierno de Chile. Ley 20.584. https://www.leychile.cl/Navegar?idNorma $=253478$.

19. Raineri G, Oyarzún M. Potencial Impacto de la nueva legislación en la investigación biomédica en Chile. Rev Chil Enferm Respir 2013; 29 (1): 5-8.

20. Organización Mundial de la Salud. Guías operacionales para comités de ética que evalúan investigación biomédica. Ginebra 2000. http://apps.who.int/iris/bitstream/ handle/10665/66641/TDR_PRD_ETHICS_2000.1_spa.
pdf;jsessionid=18196772DB37518AEF6E42EDB88F$581 \mathrm{C}$ ? sequence $=1$.

21. Sotomayor M. Regulación de la investigación biomédica en Chile. Acta Bioethica 2008; 14 (1): 79-89.

22. Oyarzún M, Pinto CM, Raineri G, Amigo H, Cifuentes L, González M, et al. Experiencia del Comité de Ética de Investigación en Seres Humanos de la Facultad de Medicina de la Universidad de Chile y los desafíos que impone la nueva legislación chilena en la investigación médica. Rev Med Chile 2014; 142 (7): 889-95.

23. Comisión Ministerial de Investigación en Salud. CMEIS. Subsecretaria de Salud Pública. Ministerio de Salud de Chile. 2015. http://www.cecmutual.cl/web/descargas/ Acceso-excepcion-a-la-Ficha-clinica-sin-CI-para-Investigacion-Agosto27-2015.pdf.

24. Comité de Ética en Investigación con Seres Humanos. Reglamento Comité de Ética de Investigación en Seres Humanos. Resolución No 3922 de 18 nov. de 2013. Facultad de Medicina, Universidad de Chile. http:// ceish.med.uchile.cl/ley/26.1.2015\%20Reglamento\%20 Interno\%20CEISH\%20(2)\%20(1).pdf.

25. Feinholz-Klip D. Consentimiento informado en investigación con seres humanos. Revista Mexicana de Bioética 2003; 1 (1): 61-2.

26. Bateman BT, Meyers P, Schumacher C, Mangla S, Pile-Spellman J. Conducting stroke research with an exception from the requirement for informed consent. Stroke 2003; 34: 1317-23.

27. Mandal J, Parija SC. Informed consent and research. Tropical Parasitology 2014; 4 (2): 78-9.

28. Kenneth V. Consentimiento para investigación en medicina de emergencia. Revista de la Facultad de Ciencias Médicas 2015; 72 (2): 113-8.

29. Rebers S, Aaronson Nl, Van Leeuwen F, Schmidt M. Exceptions to the rule of informed consent for research with an intervention. BMC Medical Ethics 2016; 17 (9): 1-11.

30. Flory J. Proposal to conduct randomized controlled Trials without informed consent: a narrative review. J Gen Intern Med 2016; 31 (12): 1511-8.

31. Bezhold G, Farnos I. El acceso a la historia clínica en investigación observacional. Rev Esp Med Legal 2017; 44 (3): 121-30. https://www.sciencedirect.com/science/ article/pii/S0377473217300718.

32. Frunza A, Sandu A. Ethical Acceptability of using generic consent for secondary use of data and biological samples in medical research. Acta Bioethica 2017; 23 (2): 288-99. https://revistas.uchile.cl/index.php/AB/article/ view/47480. 